Rev. Elev. Méd. Véf. Poys trop., 1967, 20, I (61-65).

\title{
Différents sérotypes de Salmonella isolés au Tchad
}

\author{
M. VIGIER ef G. CHAMOISEAU \\ avec la collaboration technique de Mme EGRON
}

\begin{abstract}
RÉSUMÉ
Les auteurs à la faveur des diagnostics ef des enquêtes épıdémiologiques effectués ont contribué, au Tchad, à isoler 19 sérotypes nouveaux de Solmonella.

Ils notent la possibilité de septicérnies à 5 . dublın chez l'homme et les animaux, la grande variété de sérotypes isolés à partir de cadavres de poules mortes en présentant des signes cliniques de lyphose, l'importance de l'infestation des animaux à sang froid.
\end{abstract}

Le travail que nous présentons complète les publications précédentes sur les Salmonella du Tchad (9 et 10). II est la synthèse des résultats obtenus au cours des diagnostics de routine et des enquêtes épidémiologiques effectuées durant les années 1964-1965. Tous les sérotypes ont été déterminés par le professeur L. LE MINOR, Chef du service des entérobactéries à l'Institut Pasteur de Paris.

Nous nous proposons d'énumérer les sérotypes nouvellement isolés chez l'homme et les différentes espèces animales, de les rassembler avec ceux déjà cités dans les publications précédentes, et d'essayer d'en tirer des conclusions épidémiologiques.

Homme : Les différents sérotypes ont été isolés de coprocultures effectuées à partir de selles diarrhéiques.

S. amerfoort: 1 fois

S. kolamu: 1 fois

S. korbol nouveau sérotype : 8 (20) : b, 1,5 ; 1 fois

5. omifisan : 1 fois

S. nottingham : 1 fols

S. stanleyville : 2 fois
Bovins: La recherche systématique des Salmonella dans les ganglions mésentériques des bovins, effectuée durant les années 1959-1962 n'a pas été poursuivie. Toutefois une souche de S. dublin a été isolée d'un zébu abattu à Fort-Lamy. L'aspect de la carcasse laissait soupçonner une affection septicémique et $S$. dublin fut rencontrée dans toutes les masses musculaires. Ainsi se confirme le rôle de $S$. dublin, dans les salmonelloses bovines en Afrique Centrale; une souche avait été isolée de la moelle osseuse de vaches mortes d'accidents septicémiques accompagnés d'avortements (BOUAR, P. PERREAU).

Porcins : A partir de coprocultures nous avons identifié :

S. derby : 1 fois

S. vejle : 1 fois

Le rôle pathogène de $S$. derby n'a pas été établi; S. vejle par contre semble bien avoir été la cause d'une enzootie de "pneumoentérite » survenue dans un élevage d'Abeché. En effet elle a été isolée, associée à une souche de Pasteurello multacido. Ainsı, les deux enzooties de « pneumoentérite » que nous avons pu suivre au Tchad, 
TABLEAD NOI

\begin{tabular}{|c|c|c|c|c|c|c|c|c|c|c|c|c|c|c|}
\hline Souches & H. * & Bov. & Cap. & ov. & Por. & Lap. & cob. & Poul. & $\operatorname{Con}$. & Crap. & Var" & Iéz. & Eau & Total \\
\hline 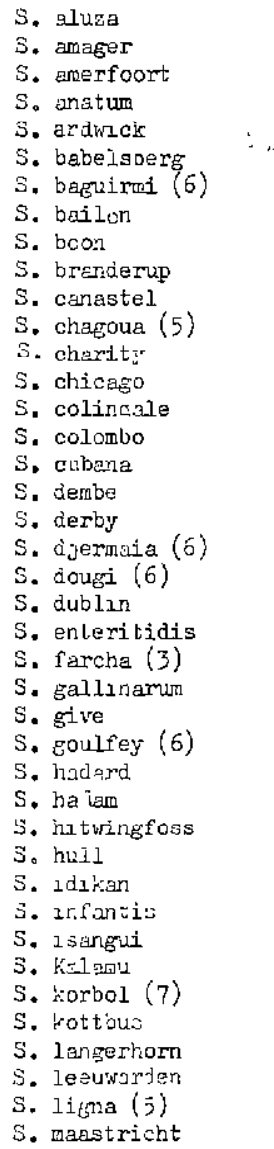 & $\begin{array}{l}1 \\
1 \\
1 \\
3 \\
2 \\
1\end{array}$ & 1 & 1 & 1 & 1 & 1 & 2 & $\begin{array}{l}2 \\
1\end{array}$ & 1 & 1 & $\begin{array}{l}1 \\
2 \\
1 \\
1 \\
1 \\
2 \\
1\end{array}$ & $\begin{array}{l}1 \\
1 \\
1 \\
1\end{array}$ & $\begin{array}{l}2 \\
2\end{array}$ & $\begin{array}{r}1 \\
5 \\
1 \\
2 \\
1 \\
1 \\
1 \\
1 \\
2 \\
3 \\
1 \\
2 \\
2 \\
2 \\
4 \\
3 \\
6 \\
1 \\
2 \\
1 \\
1 \\
7 \\
9 \\
1 \\
35 \\
1 \\
2 \\
1 \\
1 \\
1 \\
10 \\
1 \\
7 \\
2 \\
3 \\
1 \\
2 \\
1 \\
3 \\
2 \\
1\end{array}$ \\
\hline
\end{tabular}

* H. = Homene, Bov. $=$ Eovins, Cap.=Caprins, Ov.=Ousns, Por.=Porcins, Lap.$=$ Lap $\perp$ ns, Cob.=Cobaves, Poul.=Poulets, Cun $*$ Cinardş Crep. $=$ Crapauds, Var. $=$ Varans, Léz.$=$ Lézards.

mettaient en cause, l'une S. kottbus, l'autre $S$. vejle : cela met bien en doute la valeur des stocksvaccins pour cette affection.

Ovins : Par coproculture nous avons isolé une souche de S. infantis; son rôle pathogène n'a pas été établi.

Cobayes: A partir de ces anımaux de laboratoire morts pour des raisons diverses, nous avons trouvé :
S. charity : 2 fois
S. enteritidis : 1 fois
S. poona : 2 fois

Oiseaux: Des cadavres de poules reçus par notre service de diagnostic, nous avons isolé :
S. gollinarum : 9 fois

S. oranienburg : 1 fois

S. rubislow : 1 fols

S. stanleyville: 1 fois

S. typhi murium : 2 fois

S. uganda: 1 fois

Nous n'avons pu établir le rôle sûrement pathogène des Salmonella identifiées, si ce n'est pour S. typhi murium qui a été la couse d'une enzootie meurtrière dans un élevage voisin de FortLamy.

Reptiles: Nous avons poursuivi notre enquête sur l'infestation des varans (Varanus exanthematicus ef $V$. niloticus) par des salmonelles et éventuellement des shigelles. Sur 63 coprocultures 
TABLEAO NoI (suite)

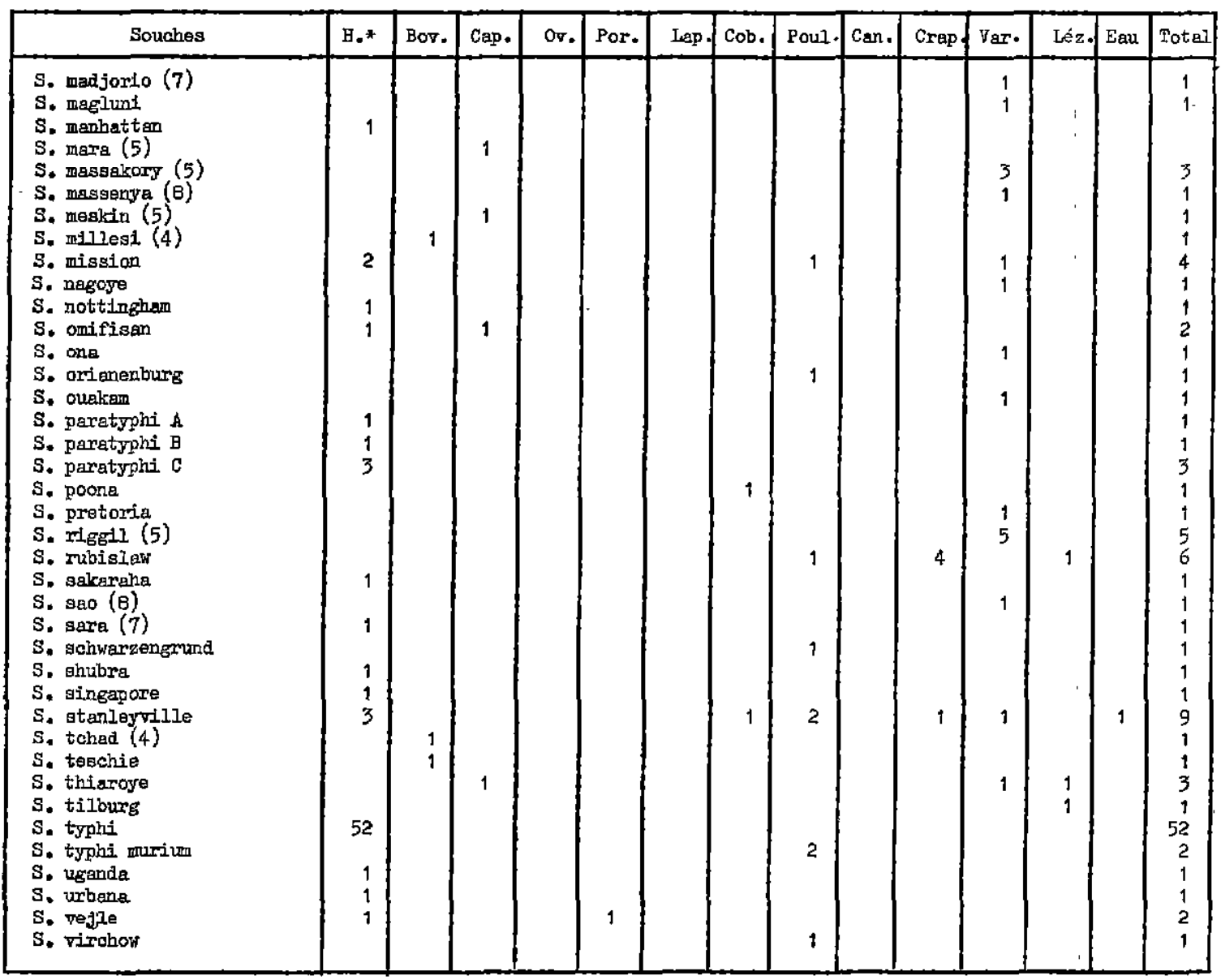

* Can.=Canards, Crep $\bullet=$ Crapauds, Var. $=$ Varans, Léz,=Lézards.

effectuées, trente-deux nous ont révélé la présence de Solmonella. Vingt trois sérotypes différents ont été déterminés, deux sont en cours de détermination.

S. aluza: 1 fois

S. cubana : 3 fois

S. dembé (nouveau sérotype : $35: \mathrm{d}$. bis) $: 1$ fois

S. farcho: 1 fois

S. hvittingfoss : 1 fois

5. isongul : 2 fois

S. kolamu: 1 fois

S. kottbus : 1 fois

S. leeuwarden : 2 fois

5. maastricht : 1 fois

S. madjorio (nouveau sérotype : $3,10: d_{1}$ enz. 15) $: 1$ fois

S. mguloni : 1 fois
S. mossakory : 1 fois

S. massenya (nouveau sérotype : 1, 4, 12, 27 : $K, 1,5): 1$ fois

S. mission : 1 fois

S. monschaui : 1 fois

S. nogoye : 1 fols

S. ona: 1 fois

S. oulkam : 1 fois

S. prétorio : 1 fols

S. riggil : 2 fois

S. soo (nouveau sérotype : 1, 3, 19 : eh, enz 15) 1 fois

Salmonello $0: 48$; sérotype indéterminé

Solmonelio $0: 47$; sérotype indéterminé.

D'autre part nous avons voulu préciser le rôle évenfuel des lézards (Agama agama) dans la contamination de l'eau destinée à la consom- 
mation humaıne; en effet, à plusieurs reprises, des cadavres de ces reptiles ont été trouvés dans les châteaux d'eau et dans les puits. Sur 61 lézards examinés, nous avons rencontré huit porteurs de Salmonella et sept sérotypes différents ont été identifiés.
S. amager : 1 fois
S. hadar : 1 fois
S. infantis : 1 fois
S. rubislaw : 1 fois
S. teschie : 1 fois
S. thiaroye : 1 fols
S. tilburg : 1 fois
Salmonella $R: 1$ fois

Divers: A la sulte d'examens bactériologiques divers nous avons identifié les sérotypes suivants:

Fuf de poule : sans coquille souillé de matières fécales.

S. idikam : 1 fois

Farine de song :

S. vejle: 1 fois

Eoux :

S. boon : 2 fols

S. chicago : 1 fois

S. colindale : 1 fois

S. stanleyville : 2 fois.

Nous signalons d'autre part que les deux souches de $S$. boon produisaient de l'indole en eau peptonée.

\section{CONCLUSIONS}

Cette revue confirme la très grande variété des sérotypes de Salmonella pouvant être isolés au Tchad chez l'Homme et dans les diverses espèces animales. Dix-neuf sérotypes nouveaux ont été isolés au Tchad : S. baguirmi, S. chagoua, S. dembé, S. djermala, S. dougi, S. farcha, S. goulfey, S. korbol, S. ligna, S. madjorio, S. mara, S. massakory, S. massenya, S. meskin, S. millesi, S. riggil, S. sao, S. sara, S. tchad. Dans le tableau ci-joint nous avons groupé tous les sérotypes cités dans les différentes publications afin de montrer leur ubiquité. De plus nous attirons l'attention sur les points suivants :

1. - L'importance de $S$. dublin capable de donner des septicémies anımales et humaines ( 9 ef 10$)$.

2. La très grande variété des sérotypes isolés à partir de cadavres de poules mortes en présentant des signes cliniques de typhose : S. gallinarum (35 fois), S. hull, S. stanleyville, S. typhi murium (2 fois), S. anatum, S. mission, $S$. orianenburg, S. rubislaw, S. schwarzengrund, S. uganda, S. virchow. Notons toutefois que leur rôle pathogène n'a pas toujours été établi.

3. - L'importance de l'infestation des animaux à sang froid : 53 p. 100 des varans (47 sur 88), 13 p. 100 des lézards (8 sur 21), 50 p. 100 des crapauds (13 sur 25) sont porteurs de Salmanella. Il est à signaler que ces sérotypes ne sont pas particuliers aux reptiles et batraciens; la plupart d'entre eux ont été couramment isolés chez l'homme. Au Tchad, S. stonleywille, S. hull, S. infantis constituent l'exemple le plus net de cette observation.

\section{SUMMARY}

\section{Identification of different Salmonella serotypes in the Republic of Chad}

During diagnosis research or extensive epidemiological surveys carried out in Chad in 1964 and 1965 the authors identified 19 new serotypes of Solmonella.

They note a possibility of septicaemias with S. Dublin in man and animals, the wide range of varieties of serotypes from dead fowls having shown symptoms oftyphosis, and the importance of Salmonella infestation in cold blooded anımals. 


\section{RESUMEN}

Varios serotipos de Salmonelfo aislados en Chad

Efectuando diagnósticos y encuestas epidemiologicas en 1964 y 1965 en Chad, los autores aislaron 19 nuevos serotipos de Salmonella Notan que septicemias. con S. dublin pueden ocurrir en el hombre $y$ en los animales. Se encuentra una gran varıedad de serotipos aislados a partir de gallinas muertas al mostrar síntomas de tifoidea. La infestación de los anımales a sangre fría es importante

\section{BIBLIOGRAPHIE}

1. LE MINOR (L.). - Le diagnostic de laboratoire des entérobactéries. Editions de la Tourelle, 1963.

2. LE MINOR (L.). - Importance épidémiologique de la détermination des sérotypes de Salmonella. Ann. Inst. Pasteur, 1963, 104 : 670.

3. LEMINOR (L.), THOMÉ (M.), PERREAU (P.) et CHARIE-MARSAINES (Ch.). - Un nouveau sérotype de Salmonella : S. Farcha. Ann. Inst. Posteur, 1959, 97: 107.

4. LEMINOR (L.), THOMÉ (M.), PERREAU (P.) et CHARIE-MARSAINES (Ch.). - Deux nouveaux sérotypes de Salmonella : $S$. millesi et S. tchad. Ann. Inst. Pasteur, 1959, $97: 406$.

5. LE MINOR (L.), VIGIER (M.), THOMÉ (M.), CHARIE-MARSAINES (Ch.) etPERREAU (P.) - Six nouveaux sérotypes de Salmonella isolés à Fort-Lamy. Ann. Inst. Pasteur, 1963. $104: 830$.

6. LE MINOR (L.), CHARIE-MARSAINES (Ch.),
ZAJE-SATLER (J.), DELAYE (R.), BORIES (S.), PERPEZAT (A.) et SEGONNE (J.) Nouveaux sérotypes de Salmonella identifiés en 1963. Ann. Inst. Posteur, 1964, 106 : 931.

7. LEMINOR (L.), VIGIER (M.), SEGONNE (J.), PETIT (M. Th.) et COLLARD (N.). Quatre nouveaux sérotypes de Salmonella isolés au Tchad. Ann. Inst. Pasteur, 1965, $109: 445$.

8. LE MINOR (L.) et Coll. : Nouvedux sérotypes de Salmonella. Ann. Inst. Pasteur, 1966, à paraître.

9. PERPEZAT (A.), PERREAU (P.), THOMÉ (M. VIGIER (M.). - Différents sérotypes de Salmonella isolés en République du Tchad. Rev. Elev. Med. Vet. Poys trop., 1964, 17 : 35.

10. SEGONNE (J.). - - Rôle de S. typhi et des Salmonelloses au Tchad. Bull. Soc, Path. exot., 1964, $57: 997$. 